efficacy assessment [Did not attend Week 12 visit $=1$, early withdrawals $=7$ (personal choice $=2, \mathrm{AE}=2$, worsening of $\mathrm{DLE}=1$, non-compliance $=1$, pregnant=1)]. The primary endpoint was met with 13/25 (52\%, 95\% CI 3173) meeting the ML-SADDLE 20 in full-set analysis. The rates for ML-SADDLE 50 and 70 were $48 \%$ and $20 \%$ respectively. Key secondary endpoints were met (table 1). Fifty-one AEs (treatment-emergent=28, Grade $3 / 4=4$ ) were recorded. There was no worsening of BILAG or SLEDAI in patients with SLE. Trough serum ETN levels were detected in 6/23 (26\%).

Conclusions Intradermal injection of ETN substantially reduced clinical activity, met its primary, secondary endpoints and was tolerable in DLE patients who were refractory to anti-malarials and other systemic therapies. This drug warrants further development in multi-centre trials. Analyses of other imaging and histological biomarkers are ongoing and can help stratifying patients for response.

Funding Source(s): National Institute of Health Research

\section{LOW-DOSE IL-2 THERAPY RESCUES DECREASED PERIPHERAL LYMPHOCYTES IN SLE PATIENTS WITH DIFFERENT INFECTIOUS STATUS}

'Sheng-xiao Zhang*, 'Jia Wang, 'Cai-hong Wang, 'Xiao-yan Wu, ${ }^{1}$ Jing Luo, 'Guangying Liu, ${ }^{1}$ Hong-yan Wen, ${ }^{2}$ Chong Gao, ${ }^{3}$ Xiao-feng Li. ${ }^{1}$ The Second Hospital of Shanxi Medical University; ${ }^{2}$ Department of Pathology,Brigham and Womens Hospital, Harvard Medical School; ${ }^{3}$ The Second Hospital of Shanxi Medical University

\subsection{6/lupus-2019-Ism.48}

Background Systemic lupus erythematosus (SLE) is a heterogeneous autoimmune disorder characterized by autoantibody production to a variety of self-antigens. This abnormal immunological background and immune-suppressive therapies predispose SLE patients to infection. Recent studies have revealed that low-dose IL-2 not only regulates immune balance but also alleviates directly SLE disease activity. In this study, we assessed the alterations of lymphocyte subpopulations and the effect of restore the immunologic balances by low dose IL-2 in SLE patients with different infectious status.

\section{(A)}

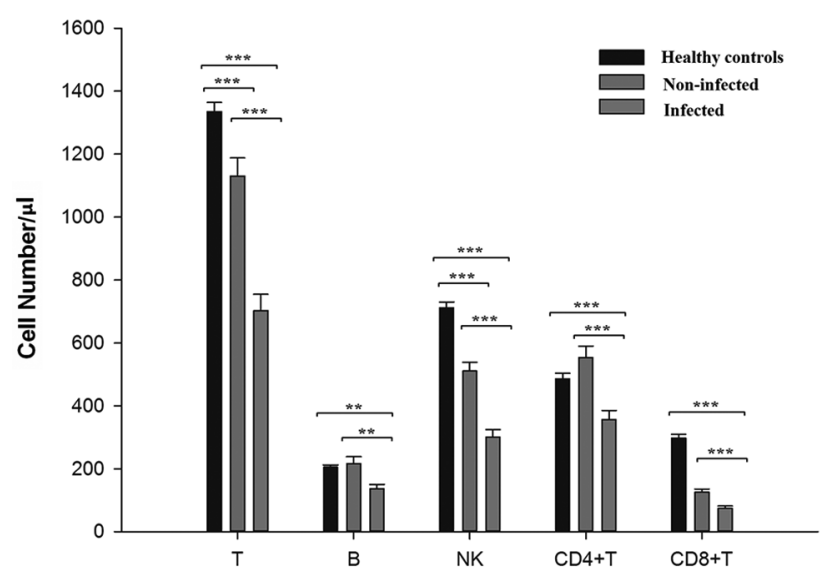

(C)

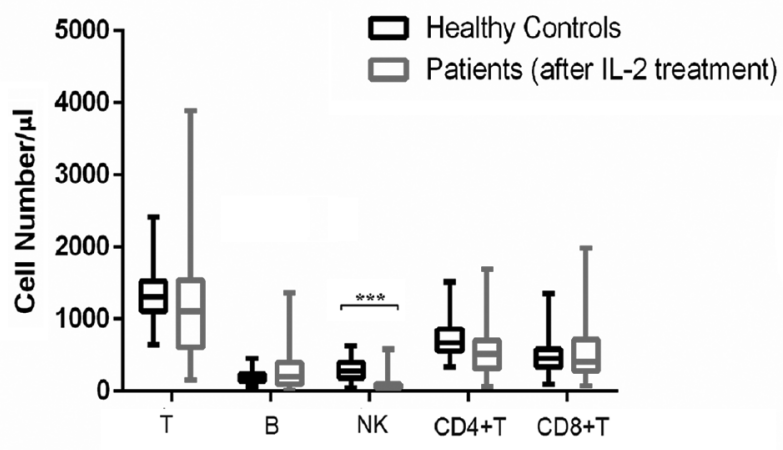

(B)

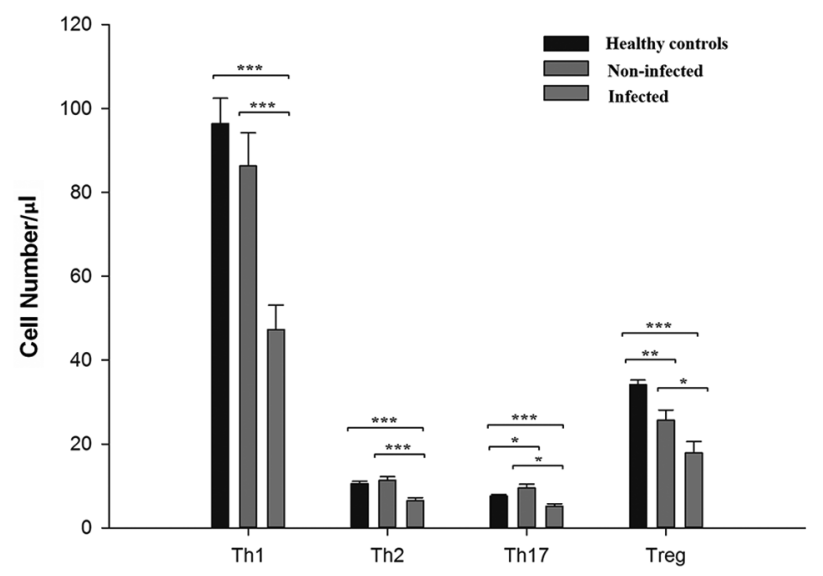

(D)

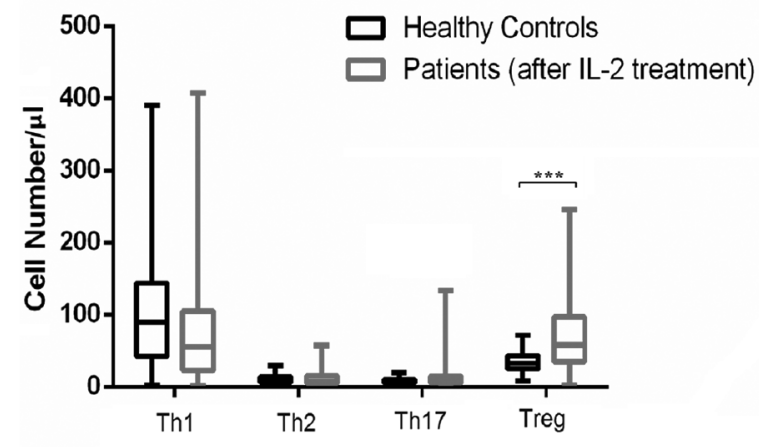

Abstract 48 Figure 1 Multiple subpopulations of lymphocytes decreased in peripheral blood of SLE patients with infection and IL-2 up-regulated lymphocytes in patients with SLE near to the levels of healthy controls. Absolute numbers of peripheral lymphocytes subpopulations were analyzed by flow cytometry. (A) SLE patients $(n=495)$ had lower levels of T, B, NK, CD4+T and CD8+T cells in PB compared with that of health controls. These cells in the infected groups $(n=162)$ were also much lower than those in non-infected patients $(n=333)$. (B) In CD4+T cells, patients with SLE had lower levels of Th1 cells, Th2 cells, Th17 cells as well as Treg cells compared with those in health donors ( $n=132)$, especially the infected groups. (C) IL-2 expanded T, B, CD4+T and CD8+T cells in PB of SLE patients $(n=54)$ even near to the levels of healthy controls $(n=132)$. (D) In CD4+T cell subsets, IL-2 restored Th1, Th2, Th17 and Tregs of SLE patients to the comparable levels of those of health donors. Importantly, Tregs were significantly increased, especially the infected groups. ${ }^{*} \mathrm{P}<0.05,{ }^{*} \mathrm{P}<0.01,{ }^{*}{ }^{*} \mathrm{P}<0.001$. 
Methods Total 495 SLE patients were enrolled. Among them, 162 with infection were determined by a positive pathogen test from various specimens or clear evidence of infection. Lymphocyte subpopulations were analyzed by flow cytometry in peripheral blood of these patients as well as 132 age-and sex-matched healthy donors. To investigate effects of low-dose IL-2 on these subsets in infection conditions, 54 patients with $(n=13)$ or without $(n=41)$ infection were received the treatment of IL-2 at 0.5 million IU per day for 5 days subcutaneously.

Results Notably, the absolute numbers of lymphocyte subpopulations in peripheral blood such as T, B, NK, CD4 + T, CD8 + T, Th1, Th2, Th17 and Treg cells in infected patients were the lowest among three groups $(\mathrm{p}<0.05$ to $\mathrm{p}<0.01)$, and non-infected patients had a higher level of Th17 and a lower level of Treg cells than those of healthy controls $(\mathrm{p}<0.05)$. Five days after low-dose IL-2 treatment, compared with the baselines before treatment, there was a significant increase in the number of studied lymphocyte subpopulations except NK cells: T $(\mathrm{p}<0.001), \mathrm{B} \quad(\mathrm{p}<0.001)$, CD4 +T $(\mathrm{p}<0.01), \quad$ CD8 + T $\quad(\mathrm{p}<0.001), \quad$ Th1 $\quad(\mathrm{p}<0.01), \quad$ Th17 $(\mathrm{p}<0.05)$ and Treg cells $(\mathrm{p}<0.01)$. Interestingly, low-dose IL2 markedly raised the absolute number of Tregs in SLE patients to an even higher level than that of healthy donors $(\mathrm{p}<0.001)$ and concomitantly, it moderately increased Th17 cells. In addition, part of patients received IL-2 therapy had a decrease in erythrocyte sedimentation rate $(\mathrm{ESR}, \mathrm{p}<0.001)$ and increased in blood routine measures such as platelet $(\mathrm{p}<0.05)$, WBC, lymphocyte and neutrophil $(\mathrm{p}<0.005)$ after the treatment.
Conclusions Patients with SLE, especially those suffering infections, had a disturbance in immune system by decreased number of various lymphocyte subsets. This preliminary finding suggests that low-dose IL-2 combination treatments restored the decreased number of lymphocyte subpopulations, neutrophils and platelets and lowered ESR of these patients. Further studies are needed to evaluate the long-term anti-infection ability of IL-2 treatment.

Funding Source(s): None

\section{CLUSTER ANALYSIS OF PATIENTS WITH SLE IN THE ADELPHI LUPUS DISEASE SPECIFIC PROGRAMME}

${ }^{1}$ Kerry Gairy*, ${ }^{2}$ Ben Hoskin, ${ }^{2}$ David Bell, ${ }^{2}$ Olivia Massey, Justyna Amelio, ${ }^{4}$ Alex Liakos. ${ }^{1}$ GlaxoSmithKline, Value Evidence and Outcomes, Brentford, Middlesex, UK; ${ }^{2}$ Adelphi Group, Adelphi Real World; ${ }^{3}$ GlaxoSmithKline, Real World Evidence and Epidemiology, Stevenage, Herts, UK; ${ }^{4} G S K$, Immuno-inflammation and Future Pipeline

\subsection{6/lupus-2019-Ism.49}

Background Organ system involvement in systemic lupus erythematosus (SLE) is highly variable. Better understanding of the symptom and organ involvement patterns in patients with SLE may facilitate making more accurate prognoses and individualized management decisions. This analysis aimed to categorize patients with SLE into clusters according to their organ system involvement.

Methods This was a secondary, descriptive analysis (208683) that utilized survey data collected from adult patients with SLE and their physicians, enrolled in the 2015 Adelphi Real

Abstract 49 Table 1 Clinical characteristics of SLE clusters

\begin{tabular}{|c|c|c|c|c|c|}
\hline & $\begin{array}{c}\text { Cluster 1: } \\
\text { Low burden } \\
n=250\end{array}$ & $\begin{array}{l}\text { Cluster 2: } \\
\text { 'Joint and skin' } \\
n=670\end{array}$ & $\begin{array}{l}\text { Cluster 3: } \\
\text { High burden; renal/hematologic involvement } \\
n=150\end{array}$ & $\begin{array}{l}\text { Cluster 4: } \\
\text { High burden; no renal involvement } \\
n=306\end{array}$ & $p$ value \\
\hline \multicolumn{6}{|c|}{ Organ systems currently affected, n (\%) } \\
\hline$n$ & 250 & 670 & 150 & 306 & \\
\hline Musculoskeletal & 0 & $659(98.4)$ & $126(84.0)$ & $306(100.0)$ & $<0.0001$ \\
\hline Mucocutaneous & $72(28.8)$ & $344(51.3)$ & $64(42.7)$ & $306(100.0)$ & $<0.0001$ \\
\hline Constitutional & $14(5.6)$ & $102(15.2)$ & $82(54.7)$ & $216(70.6)$ & $<0.0001$ \\
\hline Hematologic & $42(16.8)$ & 0 & $149(99.3)$ & $186(60.8)$ & $<0.0001$ \\
\hline Renal & 34 (13.6) & $117(17.5)$ & $87(58.0)$ & 0 & $<0.0001$ \\
\hline Cardiorespiratory & $19(7.6)$ & $39(5.8)$ & $19(12.7)$ & $46(15.0)$ & $<0.0001$ \\
\hline Neuropsychiatric & $13(5.2)$ & $33(4.9)$ & $11(7.3)$ & $32(10.5)$ & $<0.01$ \\
\hline Gastrointestinal & $3(1.2)$ & $20(3.0)$ & $12(8.0)$ & $15(4.9)$ & $<0.01$ \\
\hline Ophthalmic & $3(1.2)$ & $19(2.8)$ & $6(4.0)$ & $21(6.9)$ & $<0.01$ \\
\hline None & $74(29.6)$ & 0 & 0 & 0 & $<0.0001$ \\
\hline \multicolumn{6}{|c|}{$\begin{array}{l}\text { Number of organ systems affected, } \\
\text { mean (SD) }\end{array}$} \\
\hline$n$ & 250 & 670 & 150 & 306 & \\
\hline At diagnosis & $2.3(1.3)$ & $2.1(1.1)$ & $3.6(1.4)$ & $3.4(1.1)$ & $<0.0001$ \\
\hline Currently & $0.8(0.8)$ & $2(0.9)$ & $3.7(1.3)$ & $3.7(1.3)$ & $<0.0001$ \\
\hline \multicolumn{6}{|l|}{ Time since diagnosis (weeks) } \\
\hline $\mathrm{n}$ & 245 & 655 & 149 & 302 & \\
\hline Mean (SD) & $307.6(334.3)$ & $256.6(307.0)$ & $348.4(351.5)$ & $300.8(308.8)$ & $<0.001$ \\
\hline Current disease severity, n (\%) & & & & & $<0.0001$ \\
\hline $\mathrm{n}$ & 248 & 665 & 149 & 304 & \\
\hline Mild & $218(87.9)$ & $480(72.2)$ & $93(62.4)$ & $194(63.8)$ & \\
\hline Moderate & $27(10.9)$ & $160(24.1)$ & $46(30.9)$ & $102(33.6)$ & \\
\hline Severe & $3(1.2)$ & $25(3.8)$ & $10(6.7)$ & $8(2.6)$ & \\
\hline
\end{tabular}

SD, standard deviation 